\title{
EXTENSIONS OF TOTALLY BOUNDED PSEUDOMETRICS
}

\author{
R. A. ALO AND H. L. SHAPIRO
}

1. Introduction. The concept of extending to the topological space $X$ a continuous real-valued function defined on a subspace $S$ of $X$ has been shown to be very useful. When every continuous realvalued function on $S$ extends to $X, S$ is said to be $C$-embedded in $X$. If bounded continuous real-valued functions are considered, then $S$ is said to be $C^{*}$-embedded in $X$. Thus every completely regular Hausdorff (Tychonoff) space is $C$-embedded in the real compactification $v X$ and is $C^{*}$-embedded in the Stone-Cech compactification $\beta X$. Results in this line have been extensively studied in [5].

A similar problem is concerned with extending a pseudometric defined on a subspace $S$ to the space $X$. This problem was first studied by $F$. Hausdorff for the metric case in 1930 [6]. Hausdorff showed that a continuous metric defined on a closed subset $S$ of a metric space $X$ can be extended to a continuous metric on $X$. R. H. Bing [2] and $R$. Arens [1] rediscovered this result independently. Recently H. L. Shapiro [10] studied the problem by considering subspaces $S$ for which every continuous pseudometric defined on $S$ has a continuous pseudometric extension to $X$. Such subspaces $S$ are said to be $P$-embedded in $X$. Shapiro showed that if $S$ is $P$-embedded in $X$, then $S$ is $C$-embedded in $X$, but not conversely. However, $S$ is $P$-embedded in $X$ if $X$ is completely regular and $S$ is a pseudocompact $C^{*}$-embedded subset of $X$.

In this paper we introduce the concept of $T$-embedding, a particular case of $P$-embedding. A subset $S$ is $T$-embedded in $X$ in case every totally bounded continuous pseudometric on $S$ has a totally bounded continuous pseudometric extension to $X$. (A pseudometric is totally bounded if for every $\epsilon>0$ there is a finite subset $F$ of $X$ such that for each $x \in X, d(x, y)<\epsilon$ for some $y \in F$.) In $\S 2$ we will characterize $T$-embedding in terms of various types of open covers of the space $X$. In addition we shall show that in order for a subspace $S$ to be $T$ embedded in $X$ it is sufficient to extend every totally bounded continuous pseudometric on $S$ to a continuous pseudometric on $X$. In $\S 3$, we show that a subset is $T$-embedded if and only if it is $C^{*}$-embedded. Finally, using the concept of $T$-embedding, we give some new characterizations for a topological space to be normal.

The notation and terminology in this paper will follow that of Gillman and Jerison in [5]. Other terms used here are defined below.

Received by the editors March 27, 1967. 
Definitions. Let $\mathcal{u}=\left(U_{\alpha}\right)_{\alpha \in I}$ and $\mathcal{U}=\left(V_{\beta}\right)_{\beta \in J}$ be two families ef subsets of $X$ and let $S$ be a subset of $X$. By $u \mid S$ we mean the family $\left(U_{\alpha} \cap S\right)_{\alpha \in I}$. We say that $\mathcal{U}$ is discrete in case for each point in $X$ there is a neighborhood of the point that meets at most one member of $u$. We say that $X$ is a collectionwise normal space if for every discrete family $\mathscr{W}=\left(W_{\gamma}\right)_{\gamma \in K}$ there is a family $\left(G_{\gamma}\right)_{\gamma \in K}$ of mutually disjoint open subsets of $X$ such that $W_{\gamma} \subset G_{\gamma}$ for each $\gamma \in K$. (This definition is due to R. H. Bing [2].) If in addition $u$ is a cover of $X$ and if $v$ is a cover of $S$, then $\mathcal{U}$ is an extension of $v$ if $I=J$ and if $U_{\alpha} \cap S=V_{\alpha}$ for all $\alpha \in I$. By the star of $S$ with respect to $u$ we mean $\cup\left\{U_{\alpha}: \alpha \in I\right.$ and $\left.U_{\alpha} \cap S \neq \varnothing\right\}$. We denote the star of $S$ with respect to $\mathfrak{u}$ by $\operatorname{st}(S, \mathcal{u})$. If $x \in X$, then we write $\operatorname{st}(x, \mathfrak{u})$ for $\operatorname{st}(\{x\}, \mathcal{u})$. We set $\mathcal{u}^{*}=\left(\operatorname{st}\left(U_{\alpha}, \mathcal{u}\right)_{\alpha \in I}\right.$ and write $u<* v$ in case $\mathcal{u}^{*}$ refines $v$. A sequence $\left(\mathcal{U}_{n}\right)_{n \in N}$ of covers of a set $X$ is said to be a normal sequence in case $\mathfrak{u}_{n+1}<^{*} \mathfrak{u}_{n}$. A cover $\mathfrak{u}$ of $X$ is said to be a normal cover in case there exists a normal sequence $\left(\mathcal{u}_{n}\right)_{n \in N}$ of open covers of $X$ such that $\mathcal{U}_{1}$ is a refinement of $\mathcal{u}$. (This definition is due to Tukey [11].) If $\left(\mathfrak{U}_{n}\right)_{n \in N}$ is a normal sequence of open covers of a space $X$ and if $d$ is a pseudometric on $X$, then $d$ is associated with $\left(\mathcal{U}_{n}\right)_{n \in N}$ if the following are satisfied.

(1) $d$ is bounded by the identity function 1 .

(2) If $k \in N$ and if $d(x, y)<2^{-(k+1)}$, then $x \in \operatorname{st}\left(y, \mathfrak{u}_{k}\right)$.

(3) If $k \in N$ and if $x \in \operatorname{st}\left(y, \mathfrak{u}_{k}\right)$, then $d(x, y)<2^{-(k-3)}$. A pseudometric is separable if the topology generated by the pseudometric is separable.

For any real-valued continuous function defined on $X$, the set, $Z(f)$, of all points $x$ in $X$ for which $f(x)=0$ is the zero set of $f$. The complement of $Z(f)$ is called the cozero-set of $f$. The family $\mathcal{U}=\left(U_{\alpha}\right)_{\alpha \in I}$ is a cozero-set cover of $X$ if $u$ is a cover of $X$ and if each $U_{\alpha}$ is a cozeroset. A zero-set cover is defined in an analogous manner.

If $\left(Q_{1}, \cdots, Q_{n}\right)$ is a finite sequence of covers of a set $X$ and if $Q_{i}=\left(A_{i}(\alpha)\right)_{\alpha \in J_{i}}$ for each $i=1, \cdots, n$, then by $\bigwedge_{i=1}^{n} A_{i}$ we mean the family

$$
\left(A_{1}\left(\alpha_{1}\right) \cap \cdots \cap A_{n}\left(\alpha_{n}\right)\right)_{\left(\alpha_{1}, \cdots, \alpha_{n}\right) \in J_{1} \times \cdots \times J_{n}} .
$$

2. Equivalent formulations of $T$-embedding. We will now characterize $T$-embedding in terms of finite cozero-set covers, finite normal cozero-set covers, and finite normal open covers. To do this we will need some preliminary results that are interesting in themselves. These first results are worthy of note since they state the relationship between the topological structure induced by a pseudometric and the 
given topology on the space. We omit the standard proofs of 2.1 and 2.2. The proof of 2.3 appears in [11, Theorem 7.1].

Proposition 2.1. If $(X, J)$ is a topological space and if $d$ is a pseudometric on $X$ then $d$ is continuous if and only if $\Im_{d} \subset J$, where $\Im_{d}$ is the topology induced by $d$.

Proposition 2.2. Let $(X, J)$ be a topological space and let d be a continuous pseudometric on $X$. If $G$ is an open subset of $X$ relative to $\Im_{d}$, then $G$ is a cozero-set relative to $J$.

THEOREM 2.3. If $\left(\mathcal{u}_{n}\right)_{n \in N}$ is a normal sequence of open covers of a topological space $X$, then there exists a continuous pseudometric on $X$ that is associated with $\left(\mathcal{u}_{n}\right)_{n \in \boldsymbol{N}}$.

Since we will be considering totally bounded continuous pseudometrics we will now make the necessary modifications of previously known results. These will then be applied to obtain our desired result.

Proposition 2.4. Let $X$ be a topological space and let $\left(\mathcal{V}_{n}\right)_{n \in N}$ be a normal sequence of finite open covers of $X$. If $d$ is a continuous pseudometric on $X$ that is associated with $\left(\mathcal{V}_{n}\right)_{n \in N}$, then $d$ is totally bounded.

We omit the standard proof of 2.4.

Proposition 2.5. Let $X$ be a topological space, let $S \subset X$, and let $\mathcal{U}=\left(\mathcal{U}_{\alpha}\right)_{\alpha \in I}$ be an open cover of $S$. If $v$ is a normal open cover of $X$ such that $\mathcal{U} \mid S$ refines $\mathcal{U}$, then there is a normal locally finite cozero-set cover $W=\left(W_{\alpha}\right)_{\alpha \in I}$ of $X$ such that $W_{\alpha} \cap S \subset U_{\alpha}$ for each $\alpha \in I$.

The reader is referred to [10, Theorem 2.5] for the proof.

Lemma 2.6 If $\mathfrak{u}=\left(U_{\alpha}\right)_{\alpha \in I}$ is a finite normal open cover of the topological space $X$, then there is a normal sequence $\left(V_{n}\right)_{n \in N}$ of open covers of $X$ such that $\mathcal{V}_{1}$ refines $\mathcal{u}$ and such that $\mathcal{V}_{n}$ is finite for each $n \in N$.

Proof. By hypothesis, there exists a normal sequence $\left(\mathcal{U}_{n}\right)_{n \in N}$ of open covers of $X$ such that $\mathcal{U}_{1}$ refines $u$. Let $d$ be a continuous pseudometric that is associated with $\left(\mathcal{u}_{n}\right)_{n \in \boldsymbol{N}}(2.3)$. For each $\alpha \in I$, let $W_{\alpha}=\bigcup\left\{S_{d}\left(x, 2^{-3}\right): S_{d}\left(x, 2^{-3}\right) \subset U_{\alpha}\right\}$. Then $W=\left(W_{\alpha}\right)_{\alpha \in I}$ is a finite normal open cover of $X$ relative to $J_{d}$ (the topology on $X$ determined by $d$ ) such that $W_{\alpha} \subset U_{\alpha}$ for each $\alpha \in I$. A repeated application of [8, Theorem 1.2], and the observation that the covers constructed therein are finite, give us a normal sequence $\left(V_{n}\right)_{n \in N}$ of open covers of $X$, relative to $J_{d}$, such that $\vartheta_{1}$ refines $W$ and such that, for each $n \in N, \mho_{n}$ is finite. Since $J_{d} \subset J$ and since $W$ refines $\mathcal{U}$, the result now follows. 
Theorem 2.7 If $S$ is a subspace of a topological space $X$, then the following statements are equivalent:

(1) $S$ is T-embedded in X.

(2) Every totally bounded continuous pseudometric on $S$ can be extended to a continuous pseudometric on $X$.

(3) Every finite normal cozero-set cover of $S$ has a refinement that can be extended to a normal open cover of $X$.

(4) Every finite normal open cover of $S$ has a refinement that can be extended to a finite normal cozero-set cover of $X$.

Proof. (1) implies (2). This implication is immediate.

(2) implies (3). Assume (2) and suppose that $u$ is a finite normal cozero-set cover of $S$. By 2.6, there exists a normal sequence $\left(\mathcal{V}_{i}\right)_{i \in N}$ of open covers of $S$ such that $\mathcal{V}_{1}$ refines $\mathcal{U}$ and such that, for each $i \in N, v_{i}$ is finite. Then, by 2.3 , there exists a continuous pseudometric $d$ on $S$ that is associated with $\left(V_{i}\right)_{i \in N}$ and, by $2.4, d$ is totally bounded. Therefore, by (2), there is a continuous pseudometric $\bar{d}$ on $X$ such that $\bar{d} \mid S \times S=d$. Let $W^{\prime}=\left(S_{\bar{d}}\left(x, 2^{-4}\right)\right)_{x \in X}$. Since $(X, \bar{d})$ is a pseudometric space, it is paracompact, so there is a locally finite open cover $W$ of $X$ such that $W$ refines $W^{\prime}$. By $2.1,2.2$, and the fact that a locally finite cozero-set cover is normal, it follows that $W$ is a normal open cover of $X$ relative to the given topology on $X$ and one easily verifies that $w \mid S$ refines $\mathcal{U}$.

(3) implies (4). This implication follows from [9, Theorem 1.2] and 2.5 .

(4) implies (1). Assume (4). Let $\Im$ be the given topology on $X$ and suppose that $d$ is a totally bounded continuous pseudometric on $S$. For each $m \in N$ there exists a finite subset $F_{m}$ of $S$ such that

$$
W^{m}=\left(S_{d}\left(x, 2^{-(m+3)}\right)\right)_{x \in F_{m}}
$$

covers $S$.

Now consider any $m \in N$. Note that $\mathscr{W}^{m}$ is normal relative to $J_{d}$ and therefore, by (4), there exists a refinement of $\mathscr{W}^{m}$ that extends to a finite normal cozero-set cover $V^{m}$ of $X$. Then there exists a normal sequence $\left(v_{i}^{m}\right)_{i \in N}$ of open covers of $X$ such that $v_{1}^{m}$ refines $V^{m}$ and such that $\vartheta_{i}^{m}$ is finite for each $i \in N$.

Now for all $i, m \in N$, let

$$
\mathcal{U}^{m}=\bigwedge_{j=1}^{m} V^{j}
$$

and 


$$
u_{i}^{m}=\bigwedge_{j=1}^{m} V_{i}^{j} .
$$

Then for all $i, m \in N$, one easily verifies that

(i) $\mathfrak{u}^{m}$ and $\mathfrak{u}_{i}^{m}$ are finite open covers of $X$,

(ii) $u_{i+1}^{m}<^{*} u_{i}^{m}$ and $u_{1}^{m}$ refines $\mathcal{u}^{m}$,

(iii) $\mathcal{u}_{i}^{m+1}$ refines $\mathcal{u}_{i}^{m}$ and $\mathcal{u}^{m+1}$ refines $\mathcal{u}^{m}$, and

(iv) $\mathcal{u}^{m} \mid S$ refines ${ }^{\prime} w^{m}$.

Now again consider any $m \in N$. It follows from (i) and (ii) that $\left(u_{i}^{m}\right)_{i \in N}$ is a normal sequence of finite open covers of $X$. Then, by 2.3 , there exists a continuous pseudometric $r_{m}$ on $X$ that is associated with $\left(u_{i}^{m}\right)_{i \in N}$ and, by $2.4, r_{m}$ is totally bounded. By (ii) and (iv), we also have

(*) If $x, y \in S$ and if $r_{m}(x, y)<2^{-3}$, then $d(x, y)<2^{-(m+2)}$.

Define $r: X \times X \rightarrow R$ by $r(x, y)=\sum_{m \in N} 2^{-m} \cdot r_{m}(x, y)$. Then $r$ is a continuous pseudometric on $X$. Moreover, $r$ is totally bounded, for if $\epsilon>0$, let $k \in N$ such that $2^{-(k-1)}<\epsilon$. Since $r_{k}$ is totally bounded there exists a finite subset $F$ of $X$ such that $U_{x \in F} S_{r_{k}}\left(x, 2^{-(k+4)}\right)=X$. If $z \in X$ then $z \in S_{r_{k}}\left(x, 2^{-(k+4)}\right)$ for some $x \in F$ and it follows that $z \in \operatorname{st}\left(x, \mathcal{u}_{k+3}^{k}\right)$. Furthermore, if $1 \leqq m \leqq k$, then by (iii) we have $\mathcal{u}_{k+3}^{k}$ refines $\mathfrak{u}_{k+3}^{m}$ and hence $z \in \operatorname{st}\left(x, \mathfrak{u}_{k+3}^{m}\right)$. Hence $r_{m}(x, y)<2^{-k}<\epsilon / 2$, whenever $1 \leqq m \leqq k$. Then $r(x, z)=\sum_{m=1}^{k} 2^{-m} \cdot r_{m}(x, z)+\sum_{m=k+1}^{\infty} 2^{-m}$ $\cdot r_{m}(x, z)<\sum_{m=1}^{k} 2^{-m} \cdot(\epsilon / 2)+\sum_{m=k+1}^{\infty} 2^{-m}<\epsilon$.

From (*) it follows that

(**) If $x, y \in S$, if $i>3$, and if $r(x, y)<2^{-i}$, then $d(x, y)<2^{-(i-1)}$.

Define a relation $R$ on $X$ as follows:

$$
x R y \text { in case } r(x, y)=0 \quad(x, y \in X) \text {. }
$$

Observe that $R$ is an equivalence relation on $X$. Let $X^{*}=X / R$ be the quotient space of $X$ modulo $R$ and let $\lambda: X \rightarrow X^{*}$ be the canonical map. Then the formula $r^{*}(\lambda(x), \lambda(y))=r(x, y)(x, y \in X)$ determines a well-defined map $r^{*}: X^{*} \times X^{*} \rightarrow R$. One easily verifies that $\left(X^{*}, r^{*}\right)$ is a metric space, that $J_{r^{*}}$ is the quotient topology on $X^{*}$, and that the canonical map $\lambda: X \rightarrow X^{*}$ is an isometry. It follows that $r^{*}$ is totally bounded.

Let $S^{*}=\lambda(S)$. By $(* *)$ it follows that we can define a map $d^{*}: S^{*} \times S^{*} \rightarrow R$ as follows

$$
d^{*}(\lambda(a), \lambda(b))=d(a, b) \quad(a, b \in S) .
$$

Then one easily verifies that $d^{*}$ is a totally bounded pseudometric on $S^{*}$. 
Let $\mathcal{u}^{*}$ be the uniformity on $X^{*}$ generated by the metric $r^{*} \mid S^{*}$ $\times S^{*}$. Let $\mathcal{V}^{*}=\mathcal{U}^{*} \mid S^{*} \times S^{*}$ and note that $\left(S^{*}, \mathcal{V}^{*}\right)$ is a uniform subspace of $\left(X^{*}, \mathfrak{u}^{*}\right)$. Using $(* *)$ and the fact that $\lambda: X \rightarrow X^{*}$ is an isometry one easily shows that $d^{*}$ is a uniformly continuous pseudometric on $S^{*}$. Therefore, by $[7$, p. 42 , Corollary 16], there exists a uniformly continuous bounded pseudometric $e$ on $X^{*}$ such that $e \mid S^{*} \times S^{*}=d$. To show that $e$ is totally bounded, let $\epsilon>0$ and let $B^{*}=\left\{\left(x^{*}, y^{*}\right) \in X^{*} \times X^{*}: e\left(x^{*}, y^{*}\right)<\epsilon\right\}$. Since $e$ is uniformly continuous, $B^{*} \in u^{*}$ and therefore there exists $\delta>0$ such that $\left\{\left(x^{*}, y^{*}\right)\right.$ $\left.\in X^{*} \times X^{*}: r^{*}\left(x^{*}, y^{*}\right)<\delta\right\} \subset B^{*}$. Now $r^{*}$ is totally bounded, so there is a finite subset $F^{*}$ of $X^{*}$ such that $\bigcup_{x^{*} \in F^{*}} S_{r^{*}}\left(x^{*}, \delta\right)=X^{*}$. One easily shows that $\bigcup_{x^{*} \in F^{*}} S_{e}\left(x^{*}, \epsilon\right)=X^{*}$ and it follows that $e$ is totally bounded.

Define $\bar{d}$ on $X \times X$ by $\bar{d}=e \circ(\lambda \times \lambda)$. Since $\lambda$ is continuous relative to $\mathfrak{J}, \bar{d}$ is a continuous pseudometric on $X(2.1)$. Moreover, if $x, y \in S$, then $\vec{d}(x, y)=e(\lambda(x), \lambda(y))=d^{*}(\lambda(x), \lambda(y))=d(x, y)$. Therefore $\bar{d} \mid S \times S=d$. Since $\lambda$ is an isometry and since $e$ is totally bounded, it follows that $\bar{d}$ is totally bounded. Therefore (1) holds.

The proof is now complete.

3 . The equivalence of $T$-embedding and $C^{*}$-embedding for completely regular Hausdorff spaces. We shall prove this equivalence in several stages. In our final result we give several new necessary and sufficient conditions for a topological space to be normal. (Note that a normal space need not be $T_{1}$.)

Proposition 3.1 ([5, 15E.1]). Suppose that $X$ is a topological space and that $d$ is a continuous pseudometric on $X$. Then $d$ is totally bounded if and only if for each $\epsilon>0, X$ is a finite union of zero-sets of diameter at most $\epsilon$.

Lemma 3.2. Suppose that $S$ is $T$-embedded in $X$. If $f \in C^{*}(S)$, if $Z_{S}(f) \neq \varnothing$, and if $f \geqq 0$, then there exists $g \in C^{*}(X)$ such that $g \mid S=f$.

Proof. Let $f \in C^{*}(S)$ and suppose that $f \geqq 0$ and that $Z=Z_{S}(f)$ $\neq \varnothing$. Define $\Psi_{f}: S \times S \rightarrow R$ by

$$
\Psi_{f}(x, y)=|f(x)-f(y)| \quad(x, y \in S) .
$$

Then $\Psi_{f}$ is a continuous pseudometric on $S$. To show that $\Psi_{f}$ is totally bounded, let $\epsilon>0$ and choose $k \in N$ such that $f(x) \leqq(k+1) \cdot \epsilon$ for all $x \in S$. For $n=1, \cdots, k$, let

$$
Z_{n}=\{x \in S: n \cdot \epsilon \leqq f(x) \leqq(n+1) \cdot \epsilon\} .
$$

Then $\left(Z_{1}, \cdots, Z_{k}\right)$ is a finite number of zero-sets of diameter at 
most $\epsilon$ and one easily verifies that $S=\bigcup_{n-1}^{k} Z_{n}$. Since $S$ is $T$-embedded in $X$, there exists a continuous pseudometric $d$ on $X$ such that $d \mid S \times S=\Psi_{f}$. Let $g: X \rightarrow R$ be defined by $g(x)=\inf _{y \in Z} d(x, y)(x \in X)$. Then $g \in C(X)$ and $g \mid S=f$.

Theorem 3.3. Suppose that $X$ is a topological space. If $S$ is T-embedded in $X$, then $S$ is $C^{*}$-embedded in $X$.

Proof. Assume $S \neq \varnothing$ and let $f \in C^{*}(S)$. Fix an arbitrary $a \in S$, let $f(a)=\alpha$, let $g=(f \vee \boldsymbol{\alpha})-\boldsymbol{\alpha}$ and $h=-((f \wedge \boldsymbol{\alpha})-\boldsymbol{\alpha})$. By 3.2, there exist $\bar{g}, \bar{h} \in C(X)$ such that $\bar{g} \mid S=g$ and $\bar{h} \mid S=h$. Let $k=(\bar{g}-\bar{h})+\alpha$. Then one easily verifies that $k \in C(X)$ and that $k \mid S=f$.

Theorem 3.4. Suppose that $X$ is a topological space. If $S$ is $P^{\aleph_{0-}}$ embedded in $X$ (every separable continuous pseudometric on $S$ can be extended to a separable continuous pseudometric on $X$ ), then $S$ is $T$ embedded in $X$.

Proof. This follows immediately from Theorem 2.7 and the fact that a totally bounded pseudometric space is separable.

Corollary 3.5. Suppose that $X$ is a topological space. If $S$ is $C$ embedded in $X$, then $S$ is T-embedded in $X$.

Proof. Since a $C$-embedded subset is $P^{k_{0}}$-embedded ([10, Theorem 4.7]), the result follows from 3.4.

Remark. Actually, T. E. Gantner has shown that a subset $S$ of a topological space is $P^{k_{0}}$-embedded if and only if it is $C$-embedded [4].

Theorem 3.6. If $S$ is a dense $C^{*}$-embedded subset of a topological space $X$, then $S$ is T-embedded.

Proof. Let $\left(G_{1}, \cdots, G_{n}\right)$ be a finite normal open cover of $S$. Then there is a cozero-set cover $\left(U_{1}, \cdots, U_{n}\right)$ of $S$ such that $\operatorname{cl}_{S} U_{i}$ is completely separated (in $S$ ) from $S-G_{i}$ for $i=1, \cdots, n$ ([9, Theorem 1.2]). Since $S$ is $C^{*}$-embedded in $X, \operatorname{cl}_{S} U_{i}$ is completely separated (in $X$ ) from $S-G_{i}$. Hence there is an $f_{i} \in C(X)$ such that $f_{i}\left(\operatorname{cl}_{S} U_{i}\right) \subseteq\{0\}$ and $f_{i}\left(S-G_{i}\right) \subseteq\{1\}$. For each $i=1, \cdots, n$, let $Z_{i}=Z\left(f_{i}\right)$ and let $V_{i}=\left\{x \in X: f_{i}(x)<1 / 2\right\}$. We note that $V_{i} \cap S \subset G_{i}$ and $\operatorname{cl}_{X} U_{i} \subset \operatorname{cl}_{X} Z_{i}=Z_{i} \subset V_{i}$. Therefore $X=\operatorname{cl}_{X} S=\operatorname{cl}_{X}\left(\bigcup_{i=1}^{n} U_{i}\right)$ $=\bigcup_{i=1}^{n} \operatorname{cl}_{X} U_{i} \subset \bigcup_{i=1}^{n} V_{i}$ and hence $\left(V_{1}, \cdots, V_{n}\right)$ is a cozero-set cover of $X$ that, on $S$, refines $\left(G_{1}, \cdots, G_{n}\right)$. It follows that $S$ is $T$-embedded in $X$.

Theorem 3.7. Suppose that $X$ is a completely regular Hausdorff space and that $S$ is a subspace of $X$. If $S$ is $C^{*}$-embedded in $X$, then $S$ is $T$-embedded in $X$. 
Proof. Since $S$ is $C^{*}$-embedded in $X, \mathrm{cl}_{\beta X} S=\beta S$. Moreover, $S$ is $C^{*}$-embedded in $\beta S$ so, by Theorem 3.6, $S$ is $T$-embedded in $\beta S$. But $\beta S$ is a closed subset of the normal space $\beta X$, so $\beta S$ is $C$-embedded in $\beta X$ and therefore by $3.5, \beta S$ is $T$-embedded in $\beta X$. Since $T$-embedding is transitive, $S$ is $T$-embedded in $\beta X$. It follows that $S$ is $T$ embedded in $X$.

COROLlary 3.8. If $X$ is a completely regular Hausdorff space and if $S$ is a subspace of $X$, then the following statements are equivalent:

(1) $S$ is $C^{*}$-embedded in $X$.

(2) $S$ is $T$-embedded in $X$.

As an immediate result of Theorems 2.7 and 3.8 and known characterizations of a normal space we have

Theorem 3.9. If $X$ is a topological space, then the following statements are equivalent:

(1) $X$ is normal.

(2) Every closed subset of $X$ is T-embedded in $X$.

(3) For every closed subset $F$ of $X$, every totally bounded continuous pseudometric on $F$ can be extended to a continuous pseudometric on $X$.

(4) For every closed subset $F$ of $X$, every finite normal cozero-set cover of $S$ has a refinement that extends to a normal open cover of $X$.

\section{REFERENCES}

1. Richard Arens, Extension of functions on fully normal spaces, Pacific J. Math. 2 (1952), 11-22.

2. R. H. Bing, Extending a metric, Duke Math. J. 14 (1947), 511-519.

3. - Metrization of topological spaces, Canad. J. of Math. 3 (1951), 175-186.

4. T. E. Gantner, Extensions of pseudometrics and uniformities, Thesis, Purdue Univ., Lafayette, Ind., 1966.

5. L. Gillman and M. Jerison, Rings of continuous functions, Van Nostrand, New York, 1960. 360 .

6. F. Hausdorff, Erweiterung einer Homoömorphie, Fund. Math. 16 (1930), 353-

7. J. R. Isbell, Uniform spaces, Math. Surveys, No. 12, Amer. Math. Soc., Providence, R.I., 1964.

8. Kiiti Morita, On the dimension of normal spaces. II, J. Math. Soc. Japan 2 (1950), 16-33.

9. - Paracompactness and product spaces, Fund. Math. 50 (1962), 223-236.

10. H. L. Shapiro, Extensions of pseudometrics, Canad. J. Math. 18 (1966), 981998.

11. J. W. Tukey, Convergence and uniformity in topology, Princeton Univ. Press, Princeton, N. J., 1940.

Carnegie Institute of Technology and Pennsylvania State University 